\title{
Photo-graphies
}

\section{Les carnets de voyage de Roland et Sabrina Michaud}

\section{Monique Sicard}

\section{(2) OpenEdition}

1 Journals

\section{Édition électronique}

URL : http://journals.openedition.org/genesis/1497

DOI : 10.4000/genesis. 1497

ISSN : 2268-1590

\section{Éditeur :}

Presses universitaires de Paris Sorbonne (PUPS), Société internationale de génétique artistique littéraire et scientifique (SIGALES)

\section{Édition imprimée}

Date de publication : 15 avril 2015

Pagination : 131-147

ISBN : 978-2-84050-992-9

ISSN : 1167-5101

\section{Référence électronique}

Monique Sicard, "Les carnets de voyage de Roland et Sabrina Michaud », Genesis [En ligne], 40 | 2015, mis en ligne le 18 mai 2017, consulté le 16 mai 2019. URL : http://journals.openedition.org/ genesis/1497 ; DOI : 10.4000/genesis.1497 


\title{
Les carnets de voyage de Roland et Sabrina Michaud
}

\author{
Présentés par Monique Sicard 1
}

\section{Voyageurs photographes}

Roland et Sabrina Michaud se plaisent à raconter l'histoire de cet empereur de Chine qui, souhaitant connaître l'étendue de son royaume, envoya un ambassadeur en faire le tour. Lorsqu'au bout de dix ans, l'homme revint, il reçut le titre de "voyageur ». Ainsi Roland et Sabrina Michaud se revendiquent-ils plus « voyageurs » que « photographes ».

Partis en 1960 en Afrique orientale pour une première expédition photographique, ils tournent rapidement leurs regards vers l'Asie. Quittant la France le 20 mars 1964 en voiture $4 \times 4$, ils atteignent Singapour le 21 décembre 1965 et sont de retour au mois de juillet 1968. Ce n'est qu'à leur arrivée, après un voyage d'une durée de quatre ans et demi, qu'ils prennent connaissance des milliers de négatifs noir et blanc et de diapositives Kodachrome réalisés. Avec rigueur, ils éliminent peu à peu une photographie sur deux, celles considérées comme de qualité insuffisante.

Le retour depuis Singapour est marqué, à l'automne 1967, par un arrêt prolongé en Afghanistan, pays pratiquement inconnu du monde occidental dont ils avaient, à l'aller, découvert les splendeurs. C'est ainsi, au prix d'exigences remarquables et conformément à leur volonté, qu'ils prennent place parmi les grands photographes professionnels. À l'origine membres de l'agence Rapho avant qu'elle ne soit rachetée en l'an 2000 par Hachette Filipacchi Médias, ils intègrent l'agence Akg-Images. Leurs photographies, dont certaines, telle L'Afghan à la rose, deviennent de véritables icônes, réveillent le goût du voyage chez beaucoup. Accompagnées de références aux littératures orientales, mais aussi de leurs propres textes, elles enverront sur les routes tant effectives qu'imaginaires d'innombrables amateurs de poésie et de paradis mythiques. Il n'est pas faux de dire qu'à la charnière des années soixante et soixante-dix, Roland et Sabrina Michaud ont mis une génération en mouvement.

À l'automne 1967, les voyageurs gagnent le Pamir afghan, ce doigt de l'Afghanistan pointé vers le nord-est qui s'enfonce entre le Pakistan et l'Asie centrale soviétique (aujourd'hui Tadjikistan) jusqu'à la Chine. Tandis que Sabrina se repose à Kaboul de trois années de voyage, Roland, accompagné d'un guide, remonte à cheval la vallée du Wakhan jusqu'à Tergan Korum, camp d'été des populations kirghizes, à quatre mille mètres d'altitude. En ce mois de septembre, il est surpris par les premières neiges. Alors qu'il photographie les chameaux perdus dans la montagne, une phrase des Noyers de l'Altenburg de Malraux commence à le hanter : « Redescendant du Pamir où les chameaux perdus appellent à travers les nuages 2 ... » Il apprend qu'au cœur de l'hiver, deux fois par an, les Kirghizes conduisent une caravane de chameaux depuis le camp d'hiver de Moulk Ali, à quelques heures du camp d'été, jusqu'à Khandud, chef-lieu administratif du Wakhan, situé deux mille mètres plus bas, à neuf jours de marche, pour se ravitailler en blé : celui-ci assurera leur survie pendant la saison d'hiver quand les troupeaux ne fournissent plus assez de lait.

1. Nous tenons à remercier Chantal Soler et Gilles Kagan.

2. L'ouvrage est paru en 1943 sous le titre La Lutte avec l'ange. Il sera réédité en 1948 sous le titre Les Noyers de l'Altenburg. 
Revenus en France, en 1968, Roland et Sabrina Michaud cherchent inlassablement à se documenter sur les caravanes du Pamir afghan, mais nul ouvrage, nul article et, par force, nulle photographie ne témoigne de ces longues marches sur le toit du monde. L'organisation d'un nouveau voyage photographique ne leur prend pas moins de trois années. Bénéficiant d'une autorisation exceptionnelle du roi d'Afghanistan, ils sont, au cours de l'hiver 1971, intégrés à l'une de ces caravanes. Partageant avec leurs compagnons kirghizes peines et dangers du voyage, ils réalisent un remarquable reportage que publiera en exclusivité la revue National Geographic ${ }^{3}$.

Le voyage à pied aura duré une trentaine de jours, dont dix passés au camp d'hiver. Sabrina Michaud est la seule femme qui ait jamais participé à ces lents cheminements ces caravanes étant une affaire d'hommes.

L'œuvre photographique née de ces aventures reste exceptionnelle, tant par son authenticité et sa rigueur que par ses qualités esthétiques. Roland et Sabrina Michaud furent les premiers à réaliser une couverture photographique de l'Afghanistan de grande valeur. Ils furent également les seuls photographes à participer à ces caravanes d'hiver. L'engagement avec lequel ils maintinrent, leur vie durant, les protocoles précis d'une réalisation photographique à deux têtes, accompagnée de fiches techniques et de carnets de voyage, est exemplaire. Si Sabrina réalisait les photographies des femmes dans les yourtes kirghizes, si Roland avait accès à des scènes où elle n'était pas présente, ils ont toujours signé de leurs deux noms livres, textes et images, sans que jamais l'accord de départ soit mis en cause.

L'analyse génétique trouve ses justifications dans un contexte où s'affrontent deux rythmes : celui de l'instantané photographique, celui si lent de la vie kirghize, dont la profondeur de l'attention portée aux êtres et aux choses fut la leçon du voyage. Elle rend compte du temps long des processus de la réalisation photographique effectuée dans des conditions extrêmes de froid, de fatigue, de danger, depuis son idée même jusqu'à ses publications sous forme d'articles, de livres, d'expositions. La prise de vue automatique n'occupe jamais qu'un fragment de seconde, mais l'« avant-image » s'est étendue sur cinq années, sans être jamais achevée : plus de quarante ans plus tard, des tirages photographiques sont toujours effectués à partir des Kodachromes ou des négatifs noir et blanc réalisés dans les années soixante et soixante-dix. Prenant appui sur les archives textuelles et iconiques de ces voyages, sur les entretiens approfondis conduits avec les voyageurs photographes, nous avons retracé le long processus de la conception puis de la réalisation de quelques photographies témoignant de la progression d'une caravane de chameaux cheminant, par des froids intenses, sur la rivière gelée du Wakhan, au cœur de paysages immenses.

Le dossier génétique au sens strict comprend des documents inédits : carnets de légendes (les captions books), carnets littéraires, carnet météo, négatifs noir et blanc, planches-contacts, diapositives Kodachromes, contacts couleur. Ces documents d'ordre privé ont donné lieu à des tirages de presse, des tirages de collection présentés dans des expositions, des publications photos et textes dans des revues, enfin plusieurs beaux livres de photographie sur l'Afghanistan 4 . Ils ne valent cependant que grâce aux longs dialogues que nous avons conduits avec Roland et Sabrina Michaud.

\section{Le voyage de 1967}

C'est le 18 septembre 1967 que Roland Michaud apprend que des chameaux suivent en hiver la rivière gelée. Il racontera ainsi 5 :

Depuis plusieurs jours, en apercevant des chameaux en liberté, j'interroge Mirza, le guide, sur l'existence éventuelle de caravanes. Il élude toujours ma question. Aujourd'hui, au sommet d'un col particulièrement difficile à négocier, nous faisons halte un moment pour reprendre souffle. Mirza pointe alors son index en direction d'une rivière qui déroule son ruban argenté dans la vallée : Da zamestan daryara yakh mezana, shotor az saresh ter mesha6.

3. Roland et Sabrina Michaud, "Winter Caravans to the roof of the world », National Geographic, vol. 141, n 4, 1972, p. 435-465.

4. Roland et Sabrina Michaud, L'Afghanistan, préface de Joseph Kessel, Paris, Hachette, coll. « Réalités », 1970 ; Caravanes de Tartarie, Paris, Éditions du Chêne, 1977 ; Mémoire de l'Afghanistan, Paris, Éditions du Chêne, 1980 ; Afghanistan, préface d'André Velter, Paris, La Martinière, 2002 ; «L'Asie des Tartares », Paris, Gallimard, 2011.

5. Lors d'un entretien réalisé au printemps 2014.

6. «En hiver la rivière est gelée, c'est là que passent les chameaux. » 


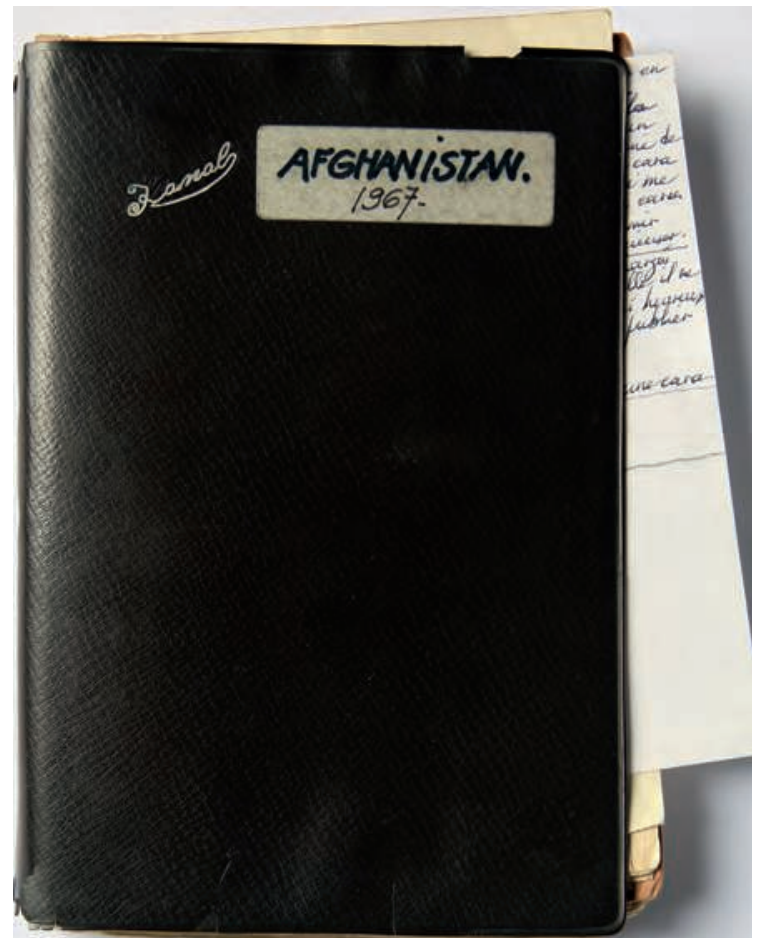

Fig. 1 : Carnet de voyage (carnet littéraire) à la couverture noire, tenu par Roland Michaud lors de son voyage à cheval de l'automne 1967 dans le Pamir afghan (coll. R. et S. Michaud)

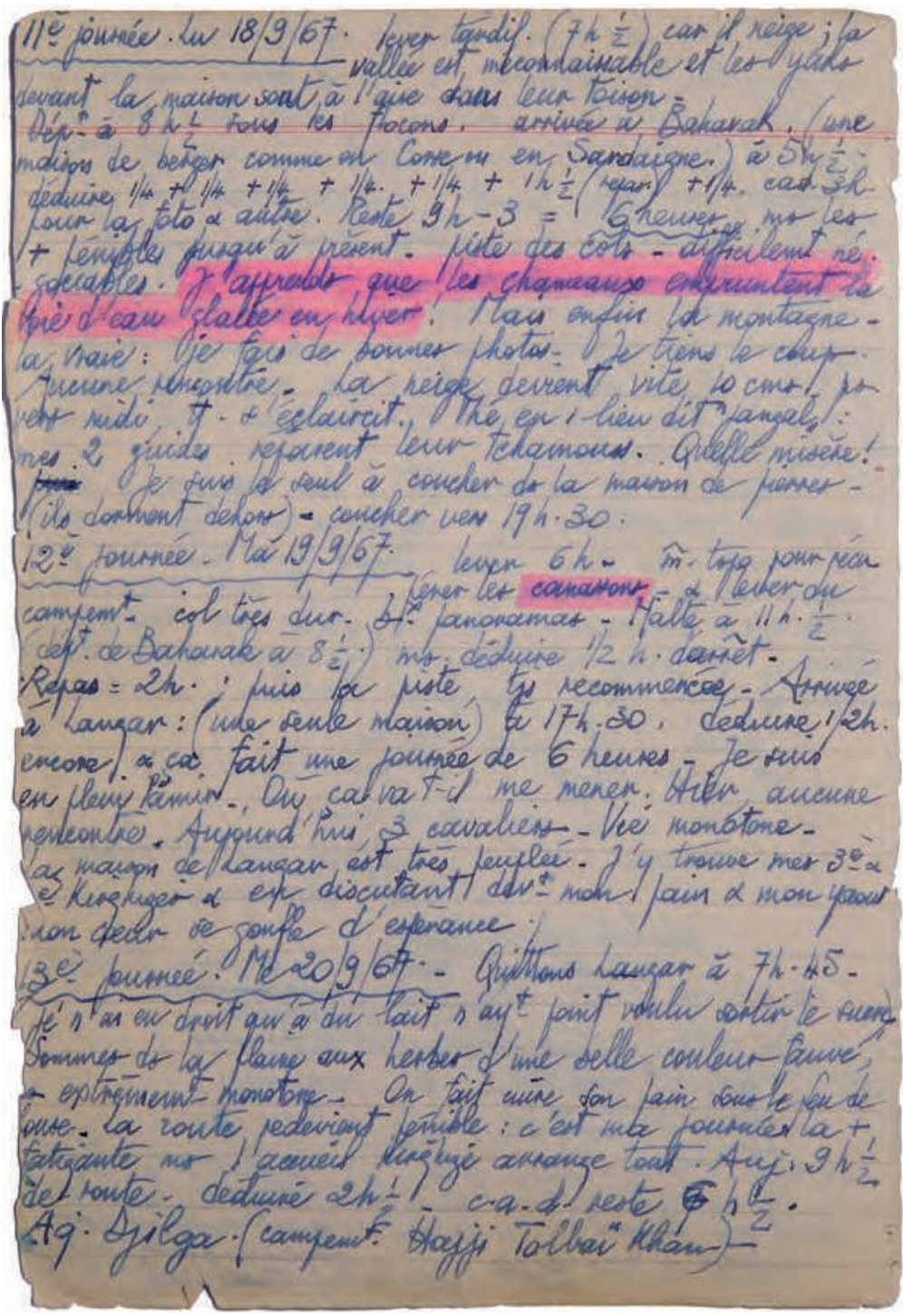

Fig. 2 : Cette page du carnet de 1967 relate les journées des 18,19 et 20 septembre 1967 , soit les onzième, douzième et treizième journées du voyage

Roland Michaud reçoit l'information comme une gifle stimulante. C'est plus tard, en préparant la seconde expédition du Pamir, qu'il surlignera dans son carnet la phrase : «J'apprends que les chameaux empruntent la voie d'eau glacée en hiver » (fig. 2), ainsi que le mot « canasson ». Il importait en effet de ne pas oublier de louer des chevaux. Le carnet fournit des indications précises sur l'emploi du temps. Sur une journée de neuf heures, Roland Michaud est à cheval six heures ; il consacre trois heures au repos et à la réalisation photographique. Le 17 septembre, il note :

Nous rencontrons des chameaux à deux bosses en liberté. Prouveraient-ils l'existence de caravanes. Où ? et quand ? et comment des chameaux lourdement chargés de marchandises pourraient-ils évoluer sur des sentes aussi étroites que celles que nous fréquentons, qui n'ont parfois guère plus que la largeur de mon pied?

Roland Michaud a atteint Tergan Korum, le camp d'été des Kirghizes. Mais alors que la frontière chinoise est très proche et que l'envie le tenaille de poursuivre, il note, le 24 septembre : "C'est fini, il faut prendre le chemin du retour »; estimant qu'agir ainsi affaiblirait l'estime qui lui a été portée par le chef kirghize, il préfère renoncer. Il espère cependant achever ses travaux sur « la caravane des deux bosses » au camp d'hiver, à seulement trois heures de là. Le photographe se montre heureux 


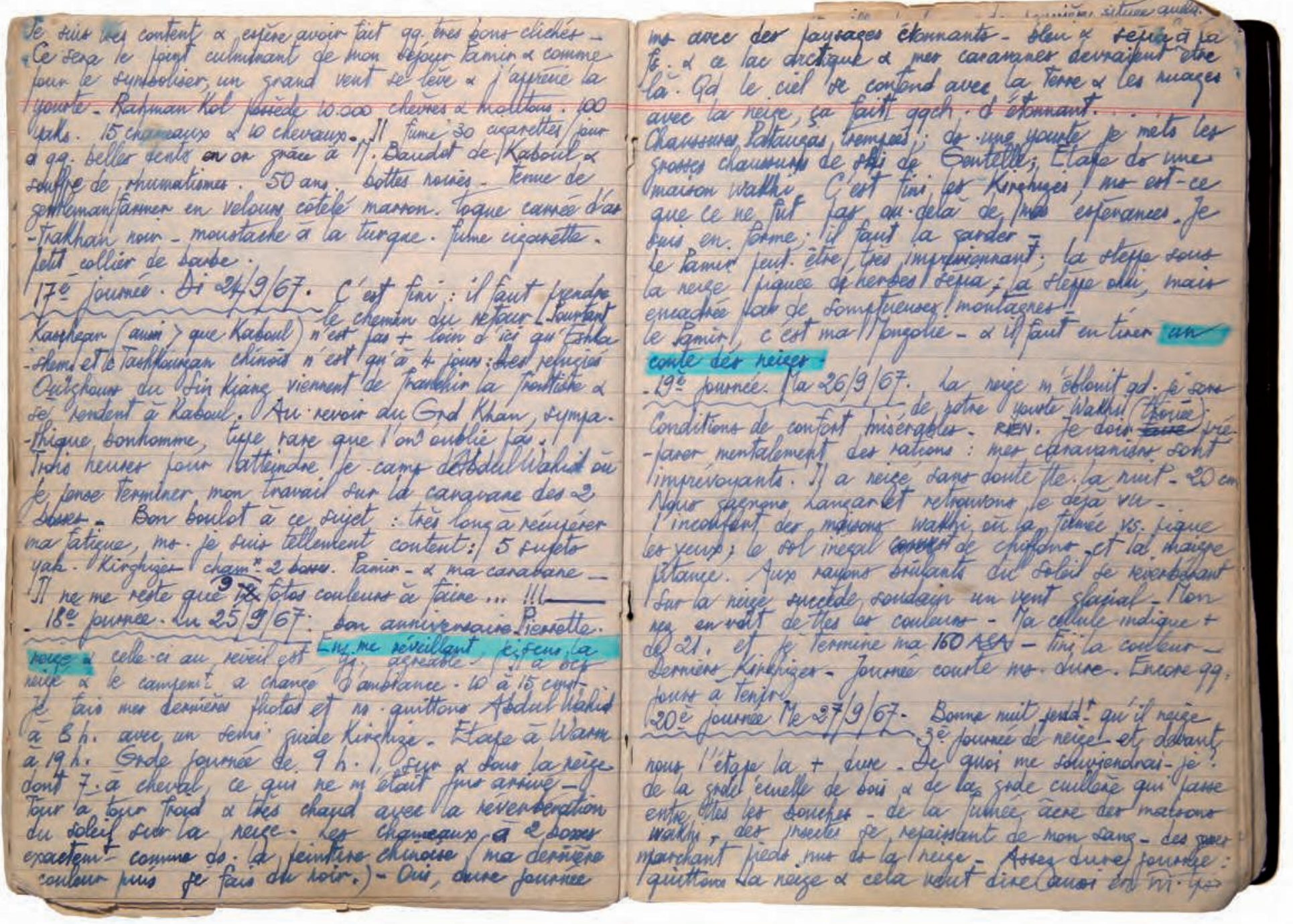

Fig. 3 : Les journées des 23 au 27 septembre 1967, ici racontées, sont respectivement les seizième, dix-septième, dix-huitième, dix-neuvième et vingtième du premier voyage dans le Pamir afghan

d'avoir pu « réaliser cinq sujets » dans cette vallée du Wakhan : les Kirghizes, le Pamir et la caravane, les chameaux à deux bosses, les yaks. Le 26 septembre, il ne lui reste que neuf photographies couleur sur sa dernière pellicule. Il remarque qu'il n'a plus que « quelques jours à tenir », même si le lendemain promet d'être la journée la plus difficile du voyage. On le sent fatigué, mais content d'avoir pu réaliser son reportage, plus heureux encore de l'accueil reçu par les Kirghizes. Le 25 septembre il écrit : «C'est fini les kirghizes, mais est-ce que ce ne fut pas au-delà de mes espérances. Je suis en forme. Il faut la garder. »

Roland Michaud a été accueilli à Tergan Korum par Rahman Qul, le grand Khan, chef des Kirghizes du Wakhan. Sa stature, son allure, son ouverture d'esprit, son accueil ont impressionné le voyageur pour lequel cette rencontre serait du même ordre que celle du moine franciscain Guillaume de Rubrouk, émissaire de Saint Louis, avec le Grand Khan de Mongolie ou même celle de Marco Polo avec Koubilaï Khan. Pour l'anniversaire de Roland, le 23 septembre, Rahman Qul organise un jeu : celui de l'arrache-chèvre. Une troupe de cavaliers se disputant sauvagement la dépouille d'une chèvre est à l'origine de certaines des plus belles photographies? L'auteur note : «Le Pamir, c'est ma Mongolie. Il faut en tirer un conte des neiges. » Ainsi se prépare tant matériellement, physiquement, humainement que dans une poétique des rêves la future grande expédition de 1971.

7. Voir les photographies publiées dans R. et S. Michaud, L'Afghanistan, op. cit., p. 146-155. 


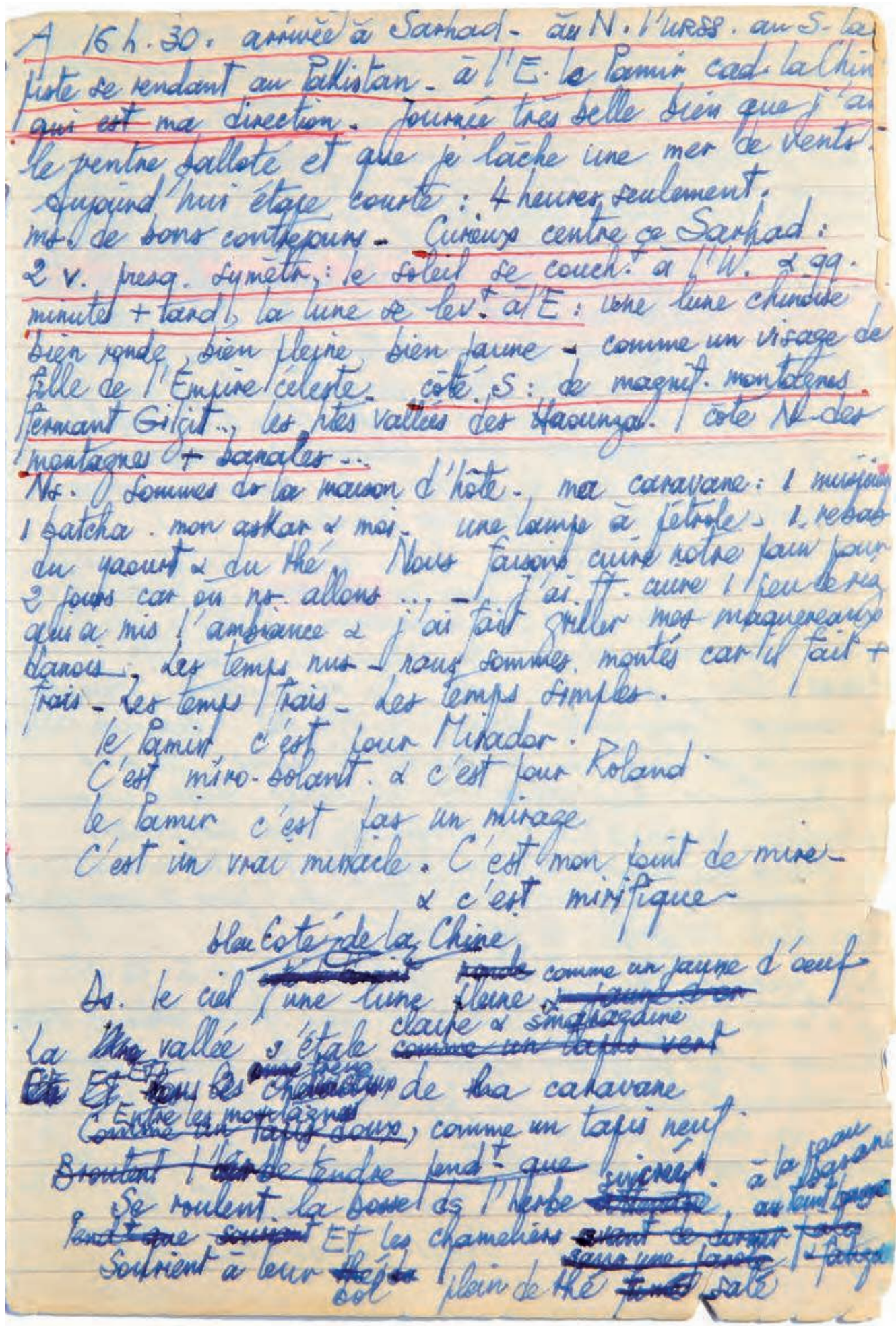

Fig. 4 : Notes rédigées le jeudi 28 septembre 1967. Lorsqu'il en a le temps, Roland écrit de courts poèmes. Ce 28 septembre, «Les temps frais. Les temps simples » témoigne de son esprit d'observation, de son humour, de son intérêt pour les mots et la littérature, de la nécessité d'exprimer la joie générée par le voyage. Redescendu des montagnes, il arrive à Sarhad. Il écrira plus tard, dans un tapuscrit personnel : «Le franchissement des deux cols avant l'arrivée à Sarhad, m'épuise. Pourtant je me souviendrai longtemps de nos deux ombres, la mienne et celle de mon cheval, et du panorama magnifique sur le corridor du Wakhan qui soudain s'offre à mes yeux. » Des passages du carnet manuscrit furent ultérieurement soulignés en rouge en vue d'une future expédition. 

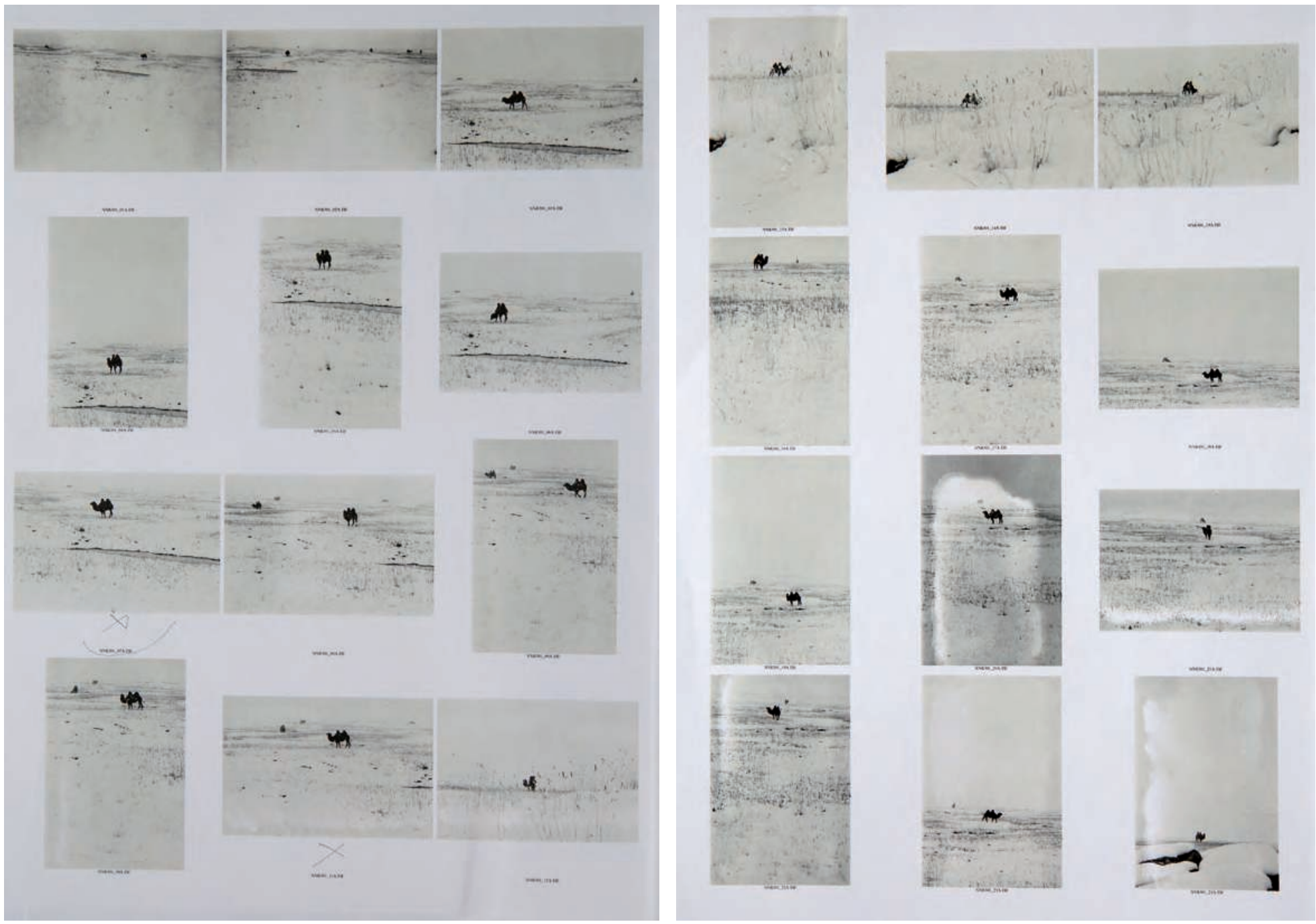

Fig. 5 : Les premières neiges donnent lieu à des photographies couleur, mais aussi à des prises de vue noir et blanc.

Les planches-contacts issues des négatifs scannés montrent que l'intérêt de l'auteur se porte plus sur l'image individuelle que sur la série

Ces deux planches-contacts (fig. 5), altérées par les années, témoignent de la rencontre de Roland Michaud avec des chameaux isolés, perdus dans les premières neiges de ce mois de septembre 1967. Le photographe, d'abord loin de son objet, se rapproche peu à peu. Il essaye différents cadrages, tourne autour d'un animal. Certains négatifs seront recadrés dans des formats carrés ou verticaux. Ces multiples essais sont le fruit des exigences esthétiques et du professionnalisme du voyageur photographe.

En 1967, Roland Michaud utilise un Pentax reflex de format $24 \times 36$. L'expédition de 1971 verra les deux voyageurs différemment outillés :
Notre équipement photographique consiste en deux boîtiers Nikon F2 appareils reflex $24 \times 36$. Nous utilisons essentiellement trois objectifs, un grand-angle $(35 \mathrm{~mm}$, ouverture F. 1,4) un petit téléobjectif ( $85 \mathrm{~mm} \mathrm{F.} \mathrm{1,8)} \mathrm{et} \mathrm{un} \mathrm{téléobjectif}$ de $180 \mathrm{~mm}$ F. 2,8. Nous travaillons avec des pellicules Kodachrome (25 et 64 ASA), quelques ektachromes plus rapides (160 ASA, qui peuvent être poussés à 320 ASA), quelques Ilford noir et blanc. Nos objectifs sont tous protégés par des filtres UV qui atténuent la dominante bleue en altitude et sur la neige. Nous n'avons ni pied photo, ni flash, ni moteur. Notre devise est simplification ${ }^{8}$.

8. Tapuscrit personnel inédit : Roland et Sabrina Michaud, « La dernière caravane. Pamir afghan 1967-1971 », 89 p. 

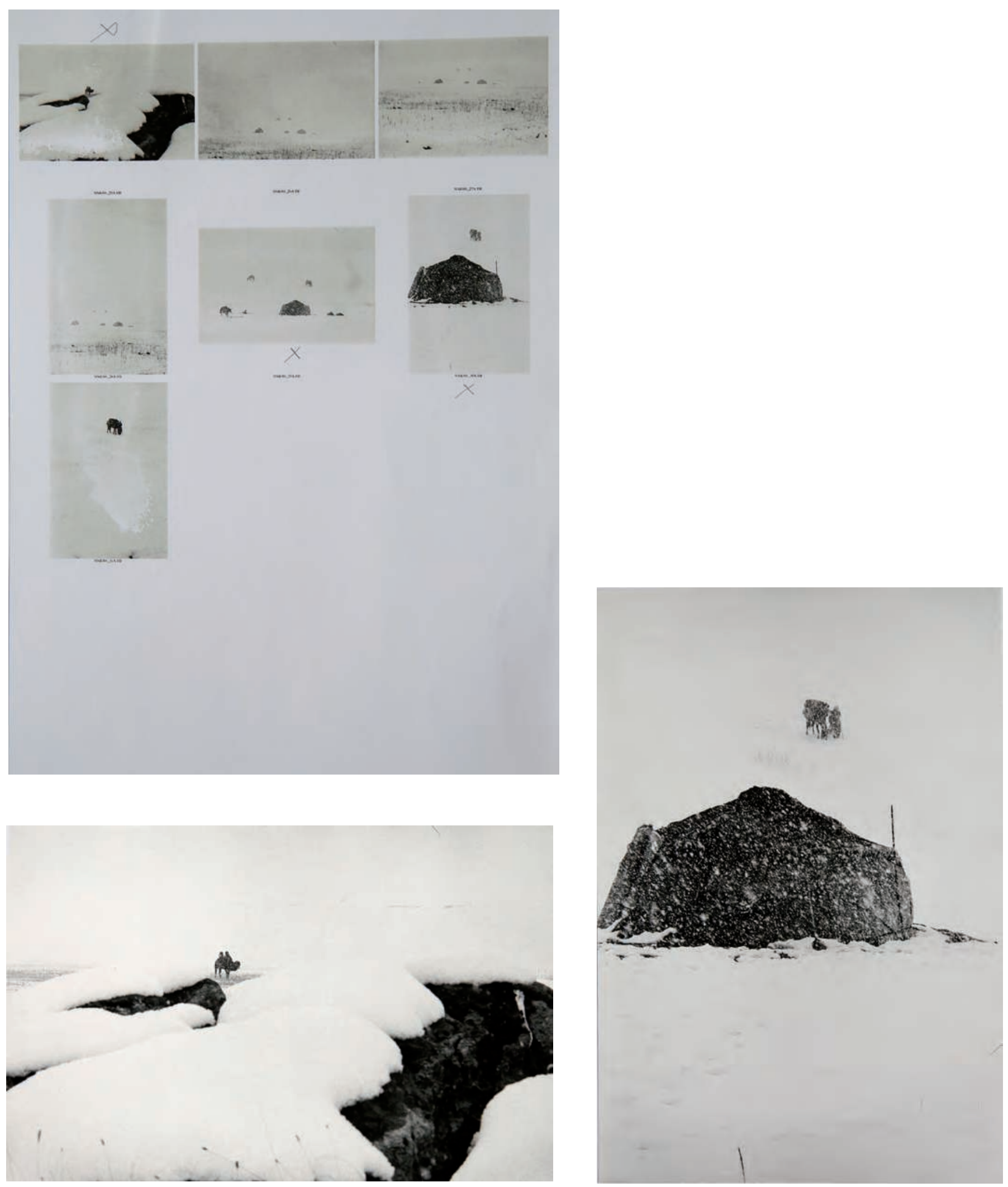

Fig. 6 : Il neige sur la haute Asie. Planche-contact et tirages de presse correspondants 


\section{Le voyage de 1971}

23 janvier 1971. Sabrina et Roland Michaud, après avoir dissimulé leur voiture dans des buissons d'épineux et l'avoir confiée aux habitants du lieu, partent à cheval rejoindre la caravane de chameaux qui a commencé la remontée vers le camp d'hiver.

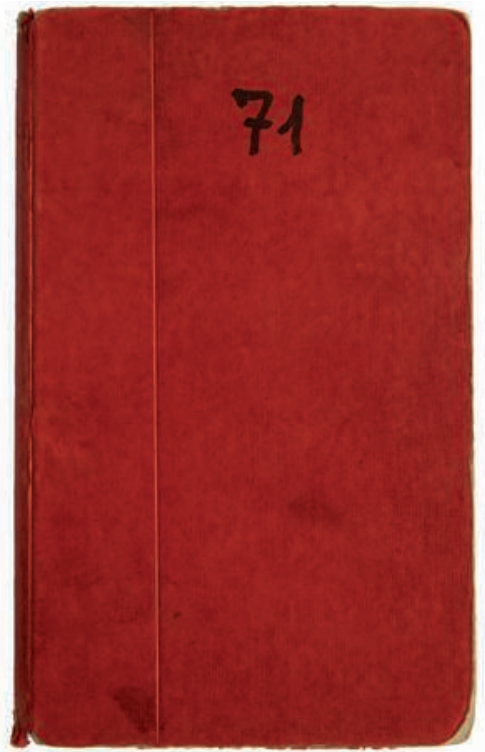

Fig. 7 : Carnet de voyage à la couverture rouge, rédigé en 1971. Ce carnet est dédicacé au fils de 2 ans de Sabrina et Roland Michaud resté en France.
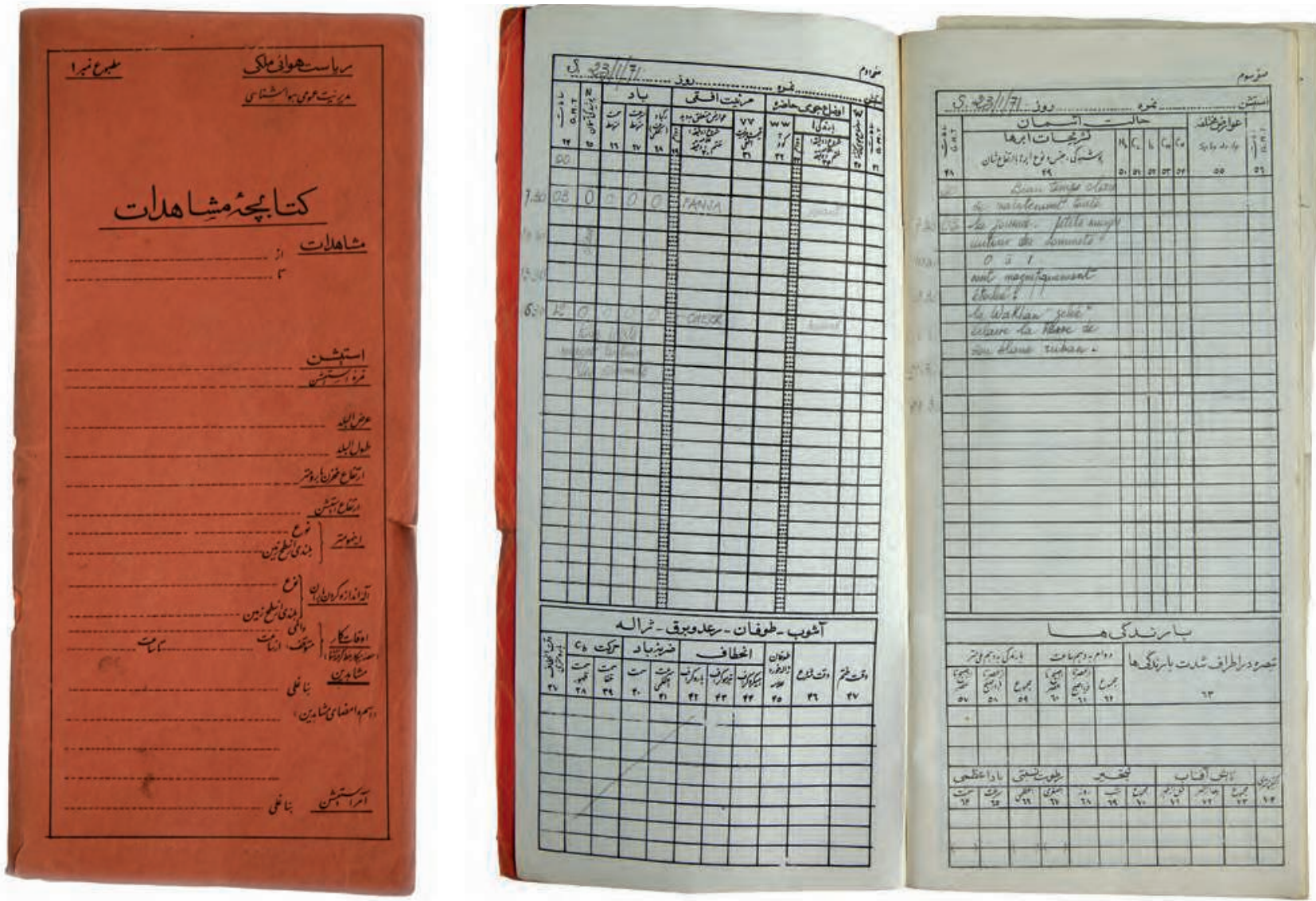

Fig. 8 : Carnet officiel de relevés météorologiques tenu par Roland Michaud

Apprenant que les voyageurs partent dans une région où aucun relevé scientifique n'a jamais été effectué, Jean Zillhardt, météorologue français en poste à Kaboul, apprend à Roland Michaud à tenir un carnet météo. Ici, les observations du 23 janvier 1971, première journée de participation à la caravane. Roland note :

« Beau temps clair», «petits nuages autour des sommets », « Nuit magnifiquement étoilée », « La Wakhan "gelée"' éclaire la terre de son blanc ruban ». 


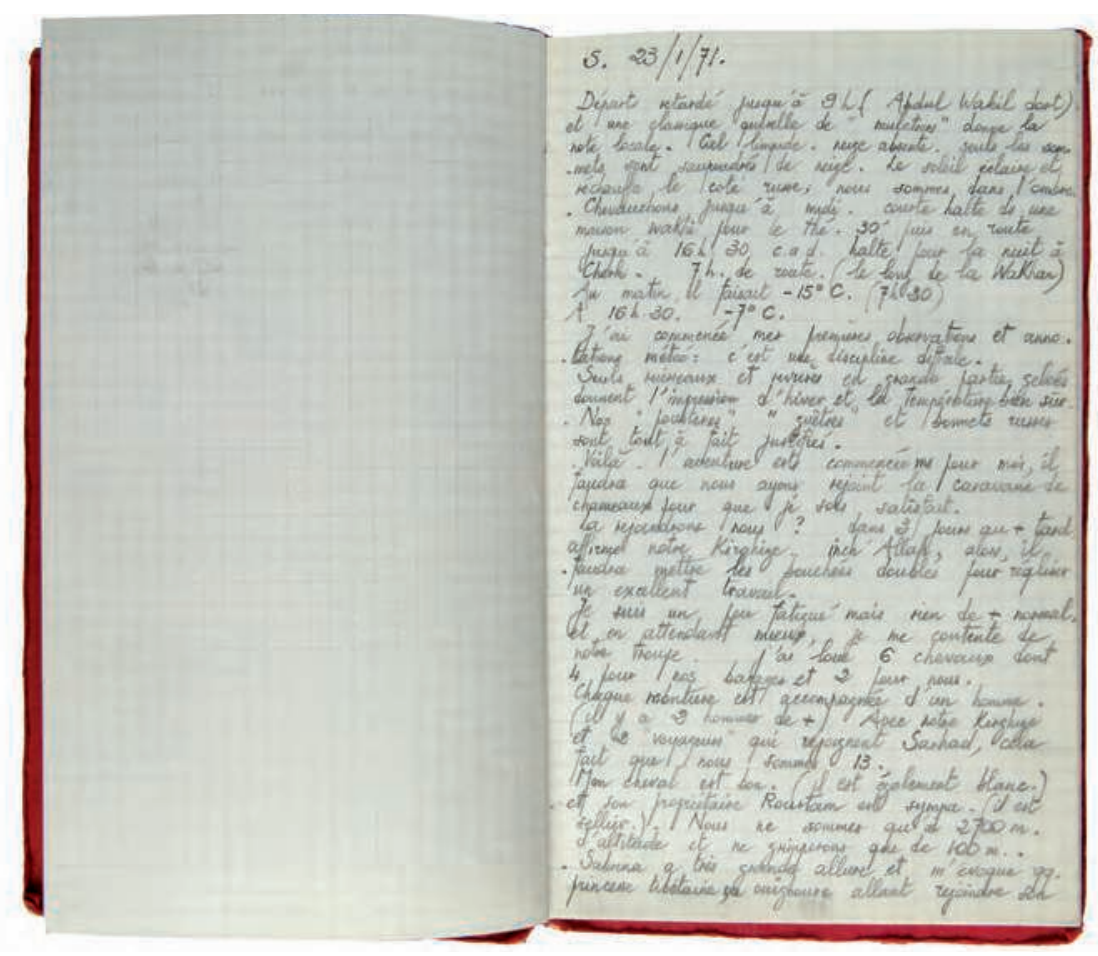

Fig. 9 : Carnet de voyage à la couverture rouge, 23 janvier 1971

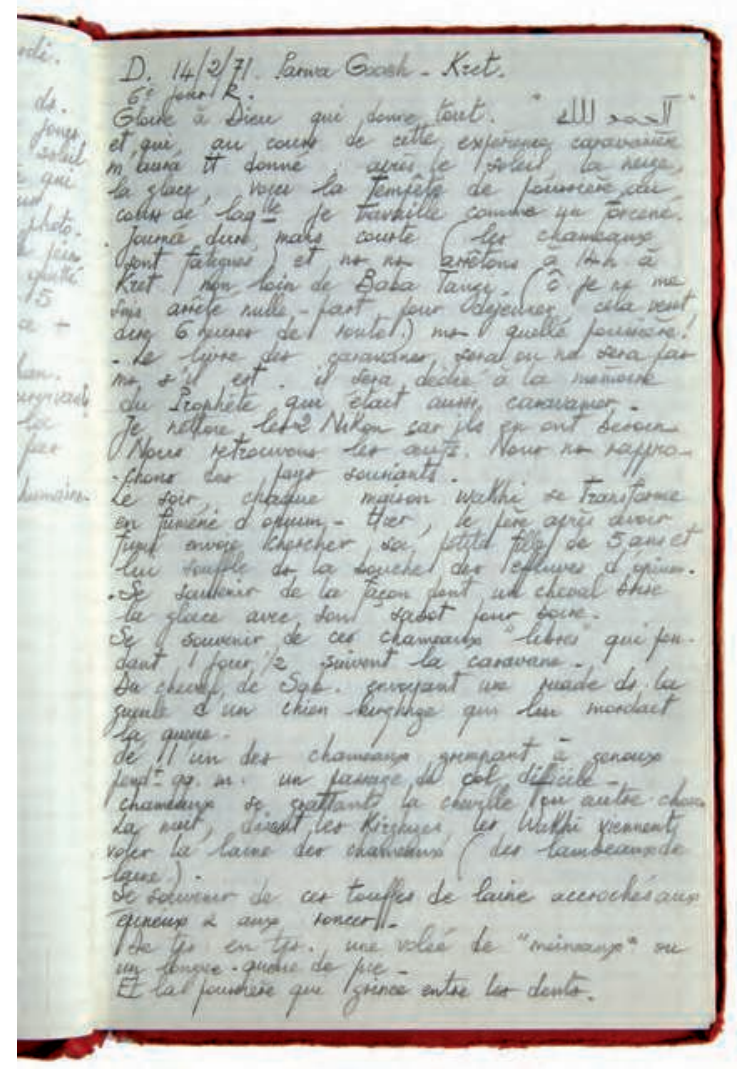

Fig. 10 : 14 février 1971, sixième jour du chemin du retour.

Arrivée à Kret, non loin de Baba Tangui, la tempête de poussière complique les prises de vue. Les voyageurs travaillent comme des forcenés. Il faut nettoyer les appareils photo et lutter contre cette poussière qui grince entre les dents et ne facilite pas les retrouvailles avec les «pays riants ». Roland songe à ce livre sur les caravanes qui reste à écrire, qui « sera ou ne sera pas ». 


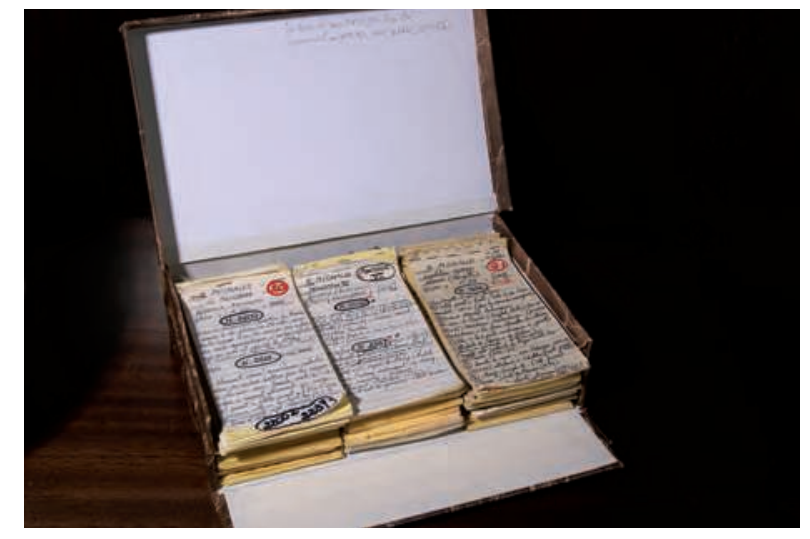

Fig. 11 : Boîte de rangement des Captions books (carnets de légendes). La réalisation de chaque pellicule, soit par Roland, soit par Sabrina, donne lieu à la rédaction d'une page dans un carnet de légendes.

\section{Roland Michaud raconte :}

De Kaboul, nous avons envoyé un télégramme au National Geographic disant que nous venions d'obtenir l'autorisation de participer à une caravane de chameaux. Les responsables nous ont donné le feu vert : «Bien sûr, allez-y ! » Ils ne se rendaient pas compte de la difficulté de la tâche.

Très rigoureux, ils nous ont donné les méthodes à suivre : nous devions documenter précisément chaque image, chaque pellicule. Grâce à eux nous avons pris l'habitude de remplir des Captions books (fig. 11). Nous avons transmis à la revue les pages originales de ces carnets rédigés au carbone et conservé avec nous les doubles, aujourd'hui peu lisibles (fig. 12 à 16).

Quand nous sommes redescendus, ils nous ont conseillé de rester sur place pour écrire sans attendre. À l'époque, le National Geographic tirait à douze millions d'exemplaires. Il y a une fiche que nous remplissions et qui est envoyée au Picture editor, au rédacteur qui s'occupe de notre sujet. Il va lire tout ce qu'il trouve sur le Pamir. Il va trouver tous les défauts et il va nous dire après : vous êtes encore des débutants car là, vous auriez dû attendre, il fallait carrément attendre. Il fallait attendre. On a beaucoup appris avec eux (fig. 17 et 18).

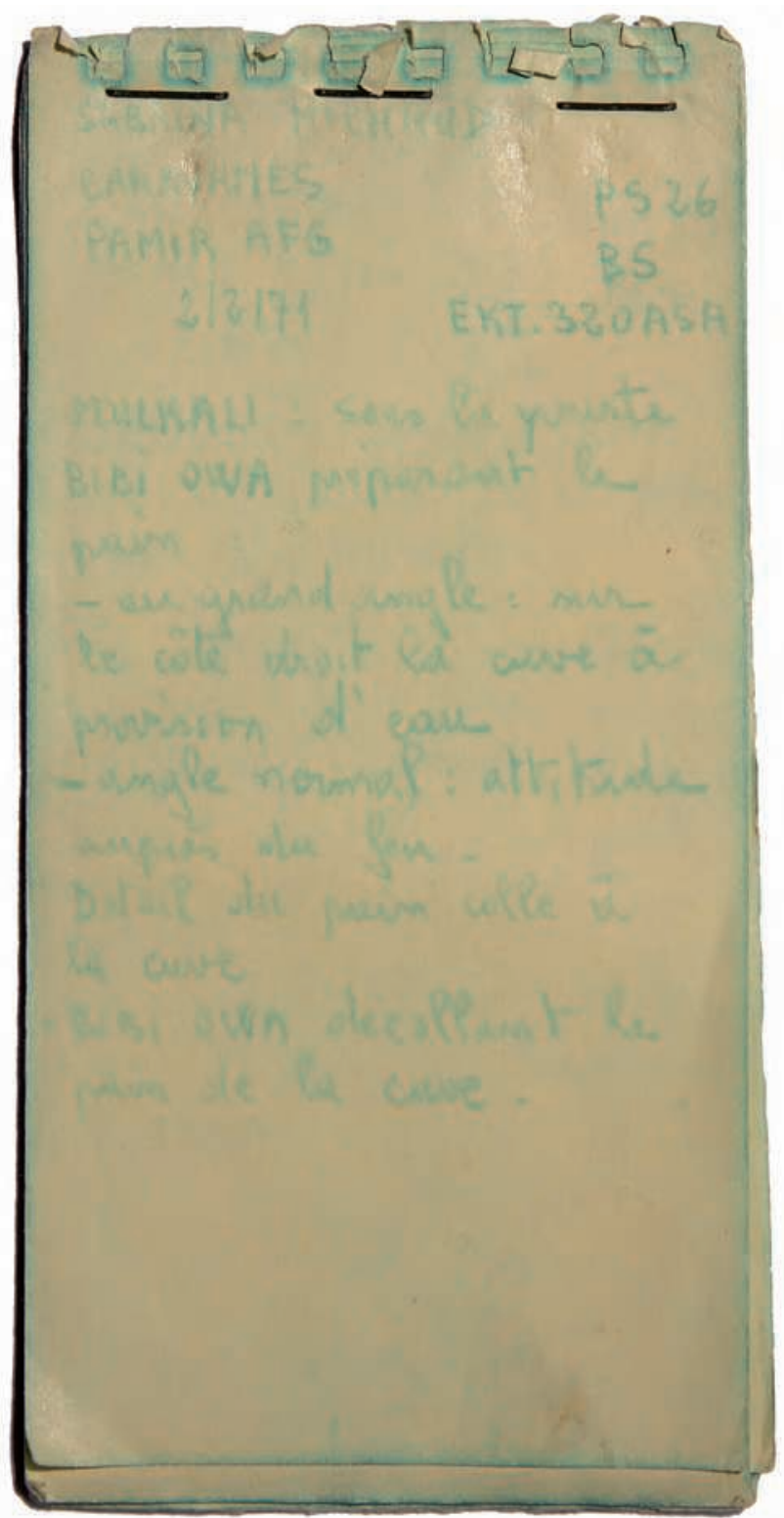

Fig. 12 : Captions book, carnet de légendes (2 février 1971)

Vingt-sixième pellicule du voyage (ektachrome) ; prises de vue réalisées par Sabrina Michaud à l'intérieur de la yourte des femmes, au camp d'hiver de Mulkali. La première page (original non visible ici) a été envoyée au National Geographic, commanditaire d'un article sur la caravane d'hiver. Il ne reste ici que l'impression carbone. 


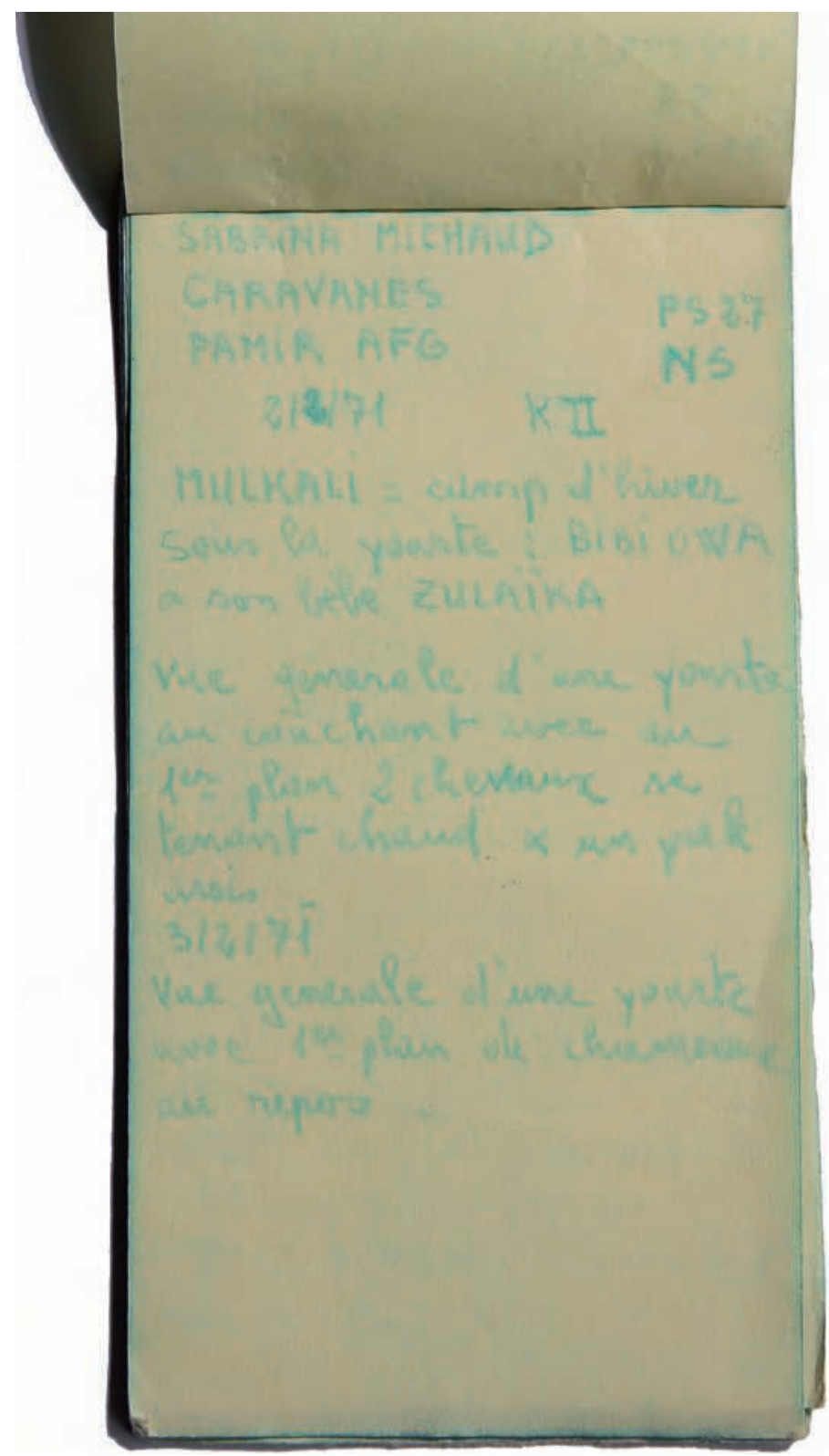

Fig. 13 : Captions book, impression carbone (2 et 3 février 1971)

Vingt-septième pellicule du voyage (Kodachrome II) réalisée par Sabrina Michaud - « MULKALI : camp d'hiver. Sous la yourte, Bibi Owa et son bébé Zulaïka. Vue générale d'une yourte au couchant avec au 1 er plan 2 chevaux se tenant chaud et un yak assis. 3/2/71. Vue générale d'une yourte avec 1 er plan des chameaux au repos. »

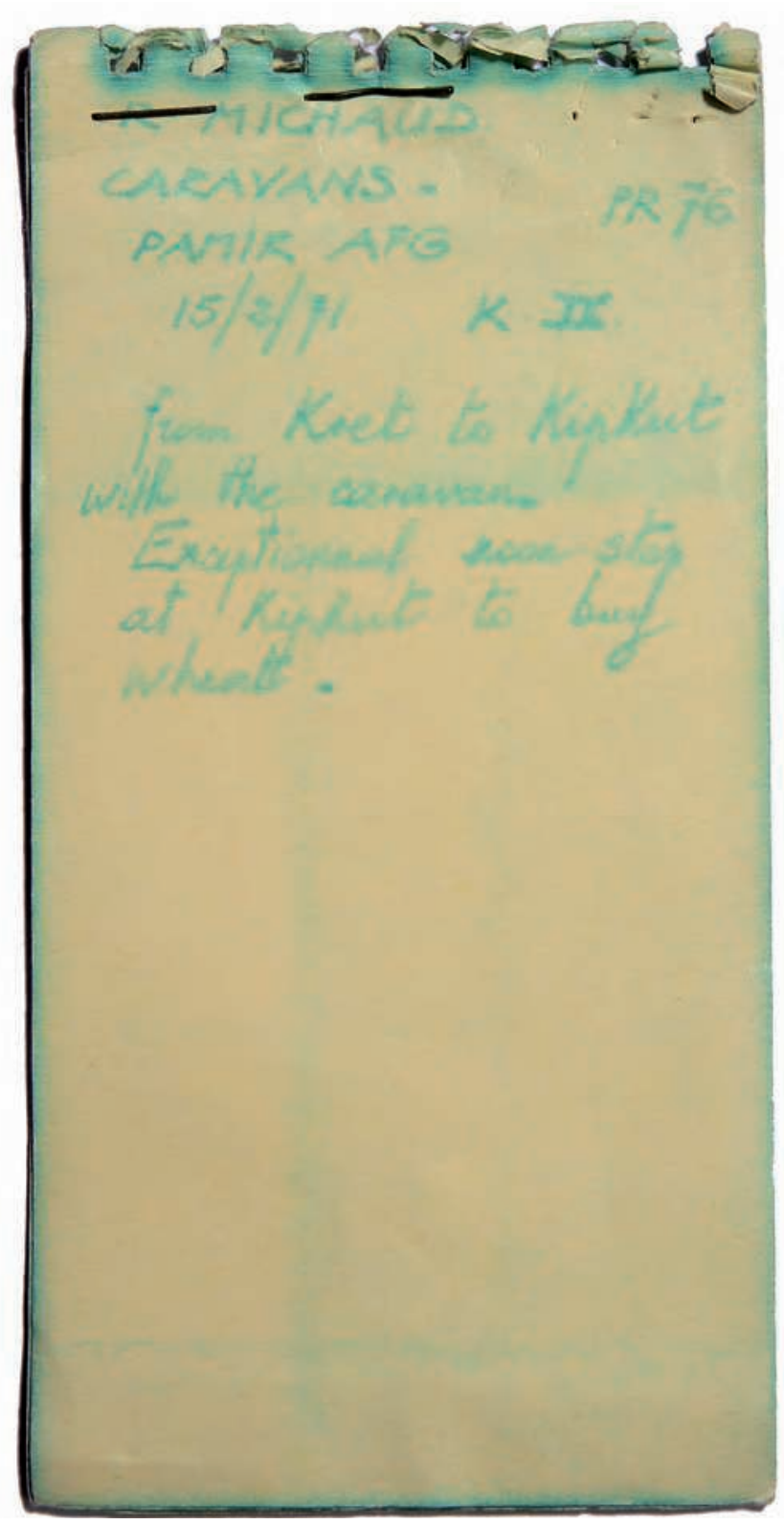

Fig. 14 : Captions book, impression carbone (15 février 1971)

Soixante-seizième pellicule du voyage (Kodachrome II). Prises de vue réalisées par Roland Michaud lors de l'étape de descente Kret-Kipkut. La caravane effectue un arrêt exceptionnel à la mi-journée pour acheter du blé : «From Kret to Kipkut. Exceptional noon stop to buy wheat. » 


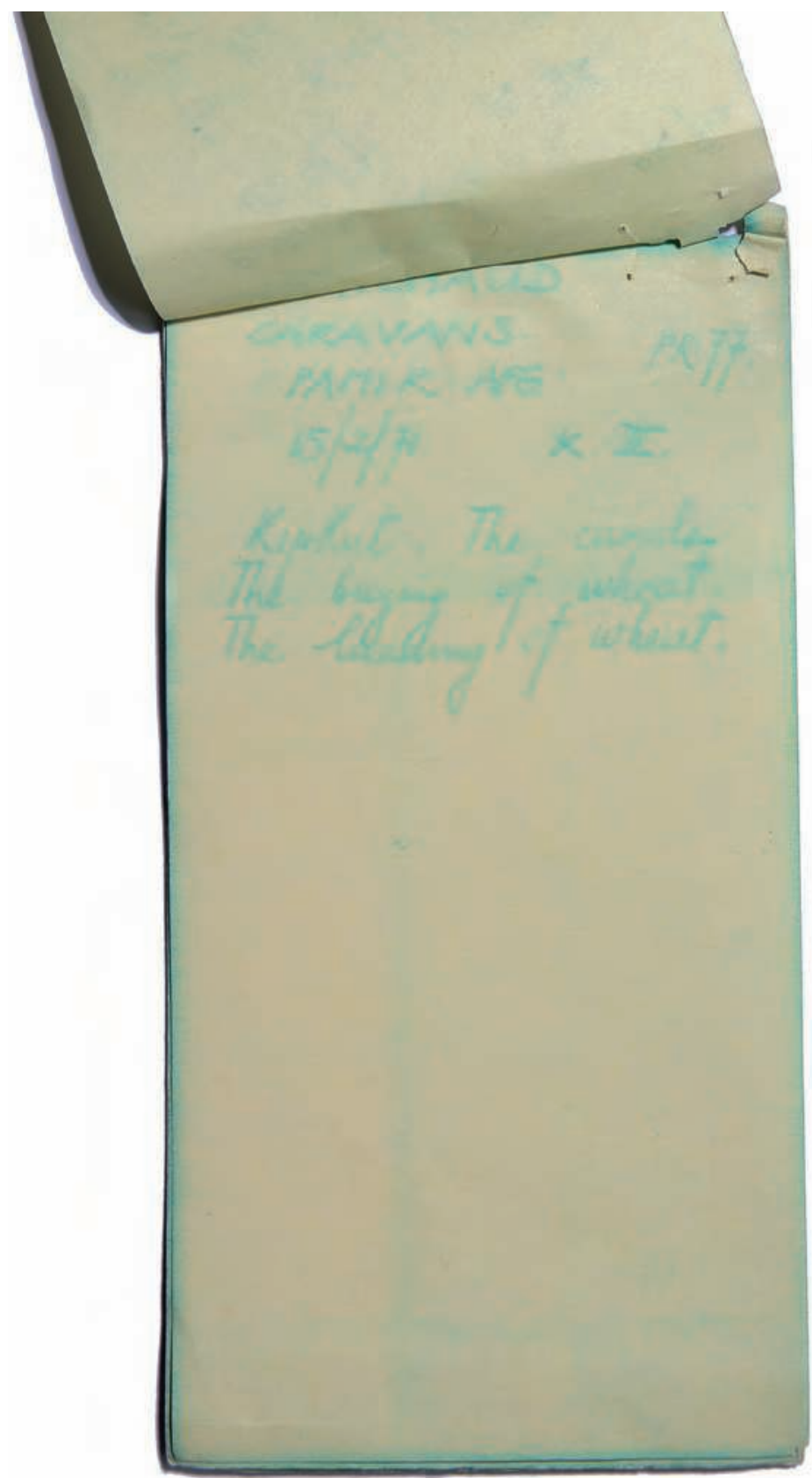

Fig. 15 : Captions book, impression carbone (15 février 1971)

Soixante-dix-septième pellicule du voyage (Kodachrome II). Prises de vue réalisées par Roland Michaud à Kipkut. Sont photographiés les chameaux, l'achat et le chargement du blé: « Kipkut. The camels. The bying of wheat. The loading of wheat. »

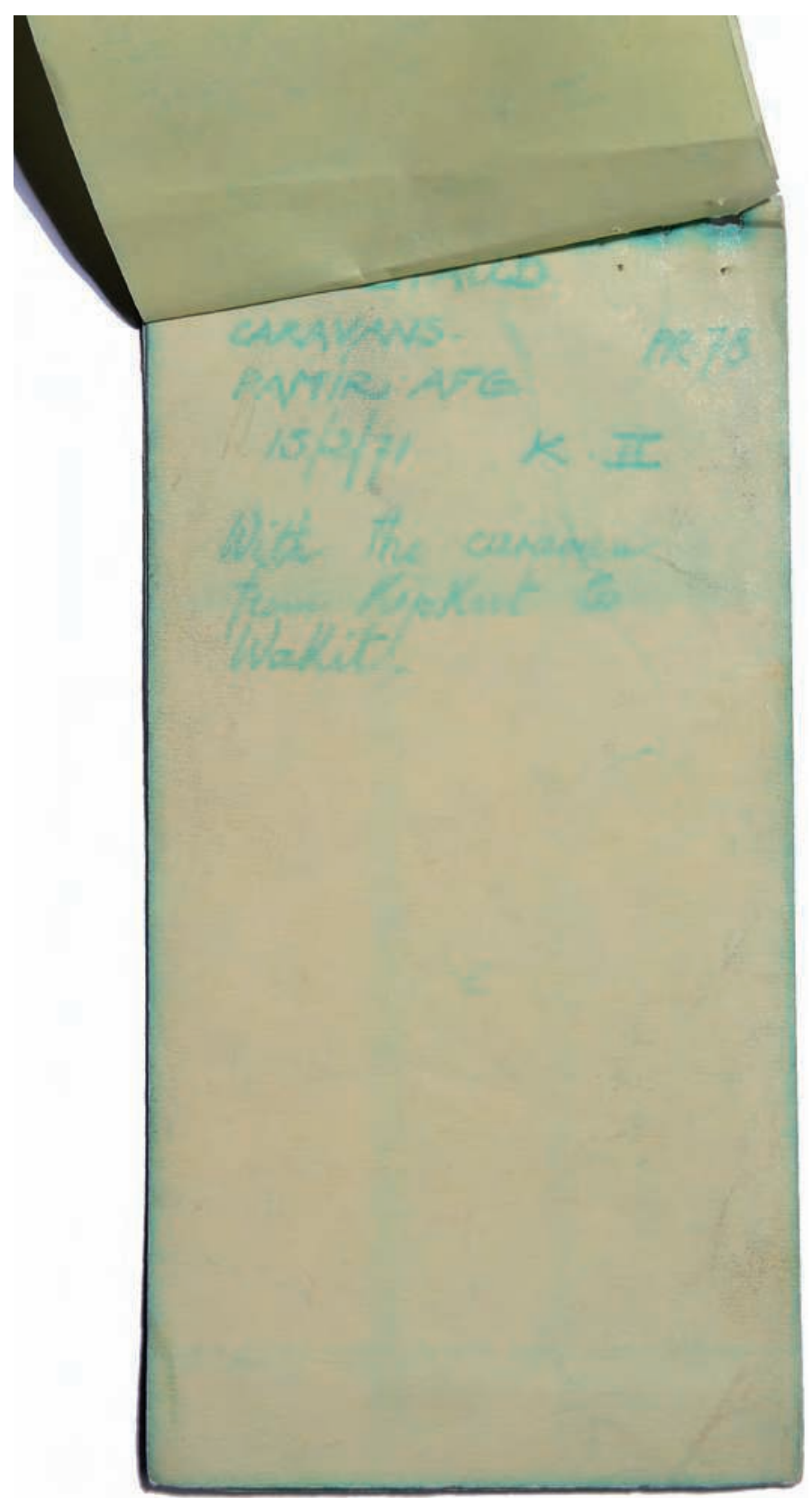

Fig. 16 : 15 février 1971. Soixante-dix-huitième pellicule du voyage (Kodachrome II)

Prises de vue réalisées par Roland Michaud : avec la caravane, de Kipkut à Wakit : « With the caravan from Kipkut to Wakit. » 


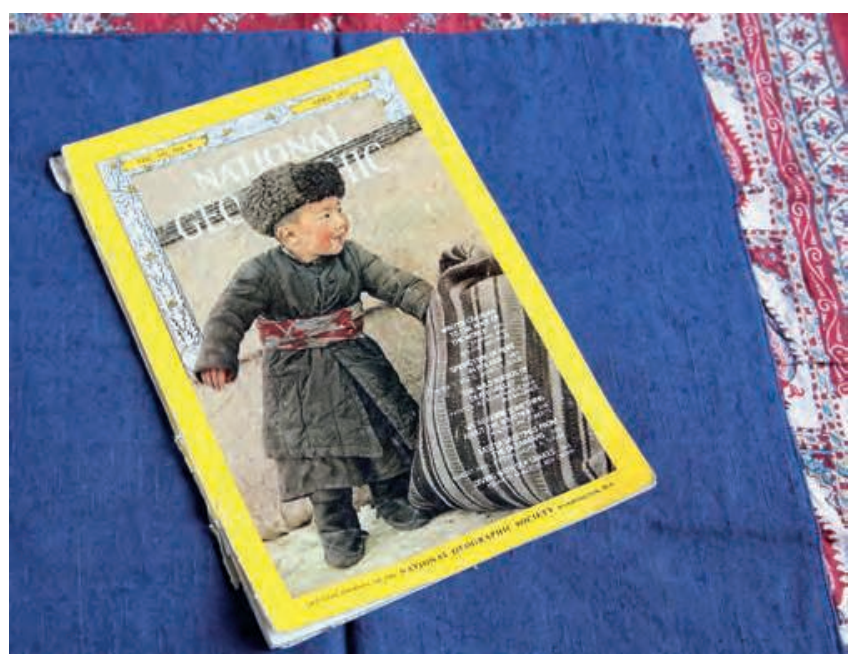

Fig. 17 : National Geographic, vol. 141, $\mathrm{n}^{\circ}$ 4. Sur la couverture, une photographie de Roland et Sabrina Michaud :

un enfant jouant malgré les très basses températures hivernales

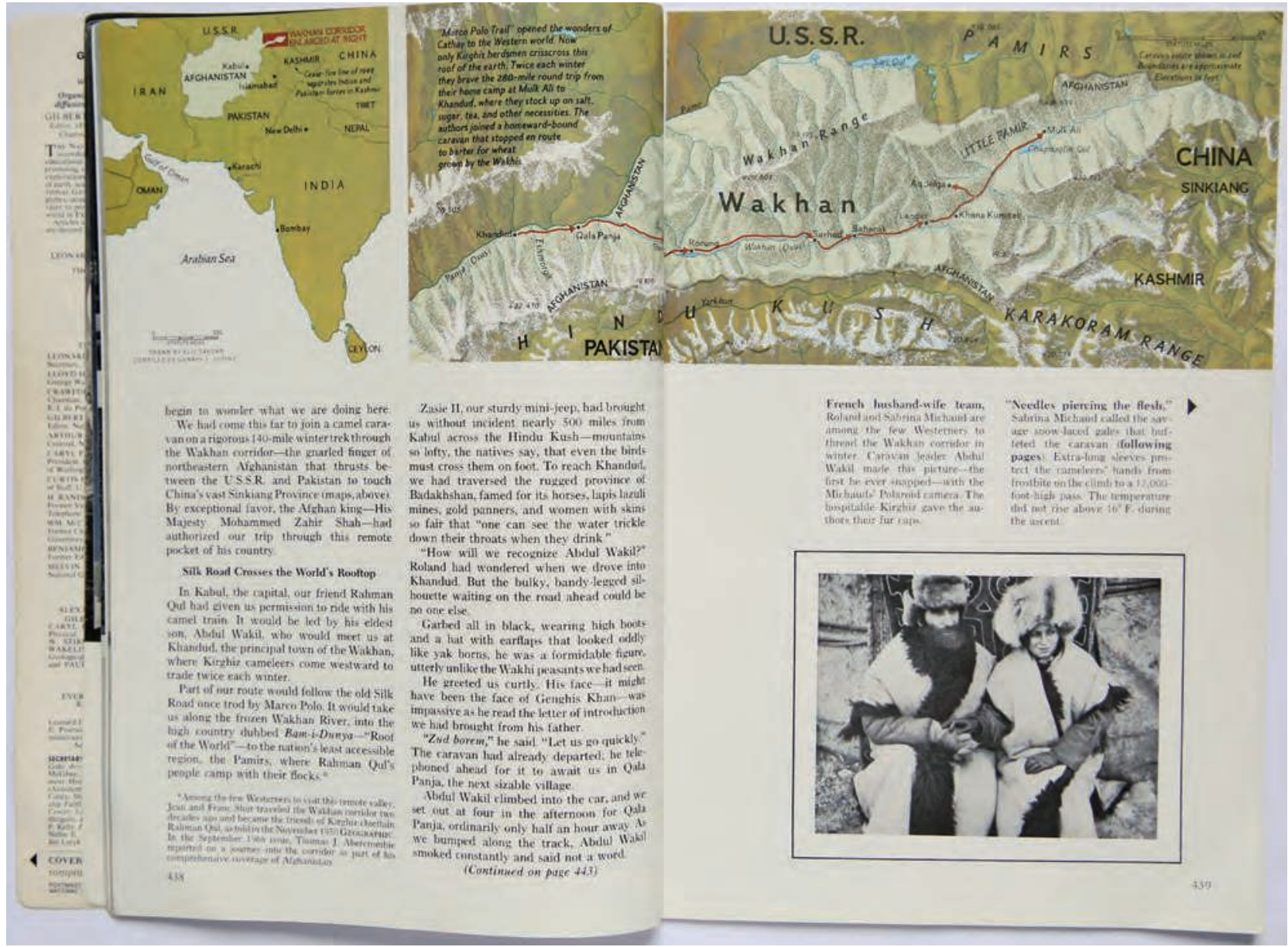

Fig. 18 : National Geographic, vol. 141, n 4. Article de Roland et Sabrina Michaud, p. 438 et 439

La carte montre le trajet suivi par les photographes et la caravane de chameaux. En médaillon noir et blanc : photographie de R. et S. Michaud réalisée par Abdul Wahil. Les photographes portent des poustines, amples vêtements traditionnels en peau de mouton retournée. Mais ici, l'absence de manches est destinée à faciliter la manipulation des appareils de prise de vue. 


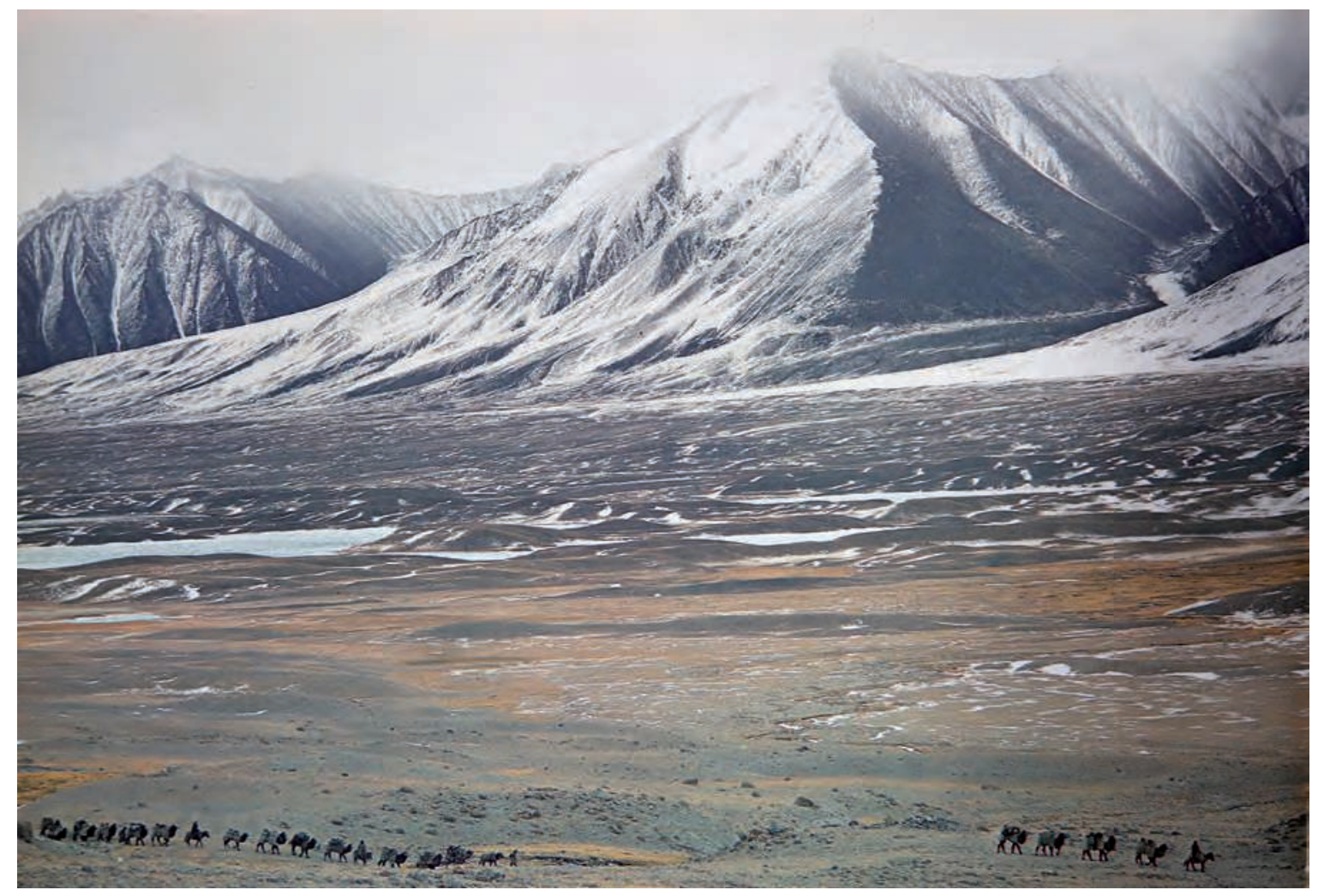

Fig. 19: La caravane (tirage couleur) Coll. R. et S. Michaud

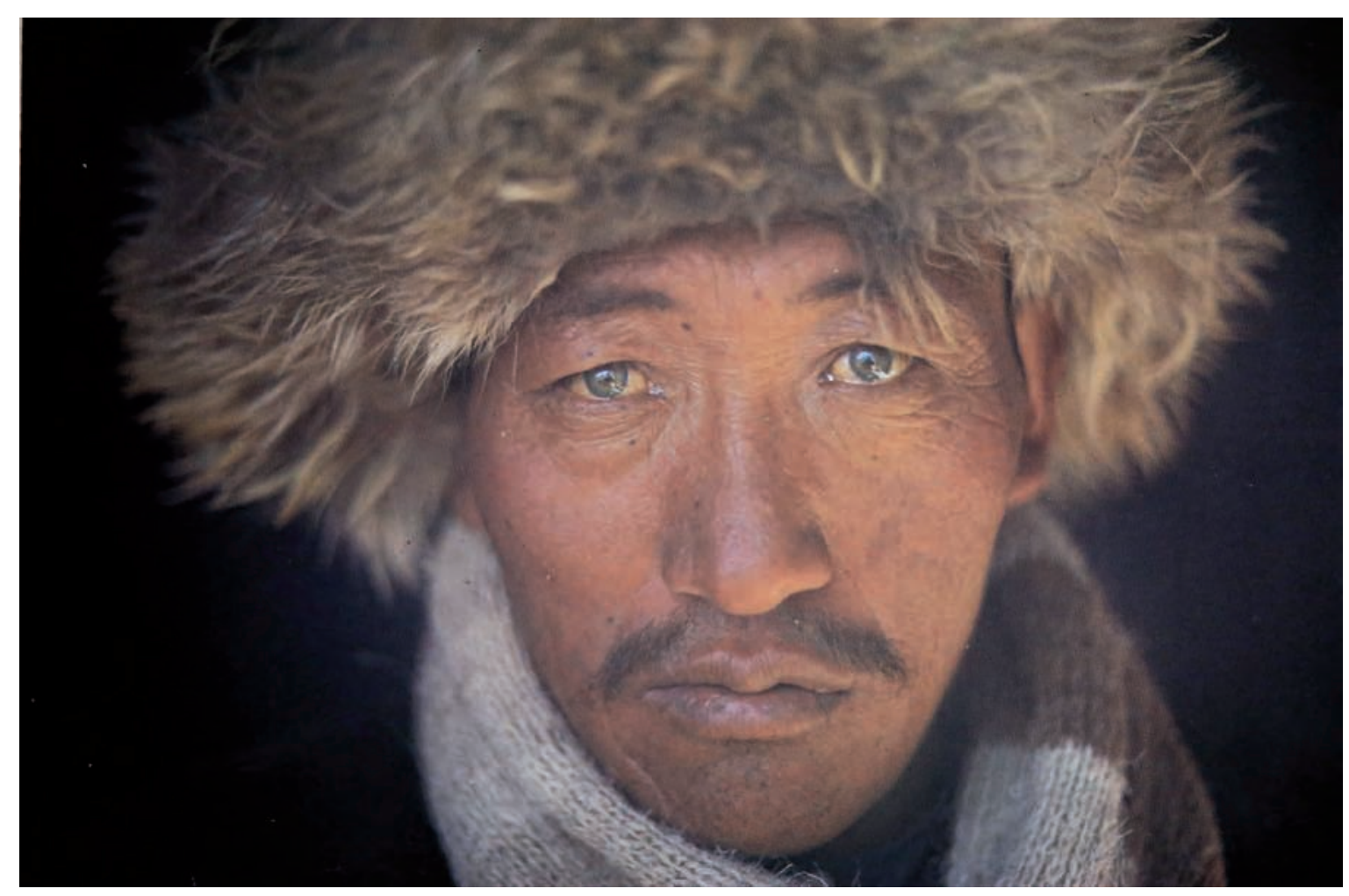

Fig. 20 : Portrait de Chatchik $^{9}$ (tirage couleur), chamelier dont les yeux gris-vert semblent refléter les teintes subtiles du paysage Coll. R. et S. Michaud

9. Parfois écrit Shahtchik par Roland et Sabrina Michaud. 


\section{Laissons parler Roland Michaud10 :}

La nourriture de base des Kirghizes est composée de laitages. Mais en hiver, à si haute altitude, le sol gelé n'offre plus assez d'herbes à brouter au troupeau pour fournir suffisamment de lait. Les Kirghizes se nourrissent alors principalement de pain et de thé.

Là réside la raison d'être de notre caravane.

Autrefois les Kirghizes se rendaient à l'est, à Kashgar aujourd'hui au Xinjiang, le grand marché du Turkestan chinois et de l'Asie centrale où ils s'approvisionnaient en thé, sucre, tissus et autres denrées de première nécessité, dont le blé, indispensable pour assurer la soudure de l'hiver. En 1950, après l'avènement de la République populaire de Chine et l'interruption du commerce, les Kirghizes du Pamir prirent l'habitude de se rendre à l'ouest, à Khandoud, chef-lieu du Wakhan où nous venions de quitter Zasie (notre voiture).

Ce blé qui pousse à trois mille mètres mûrit tard dans la saison. Il n'est transportable qu'en hiver lorsque les rivières prises dans les glaces facilitent le passage, évitant aux caravanes les hauts cols bloqués par la neige. C'est en partageant l'expérience de la vie caravanière des Kirghizes du Pamir que nous mesurons l'importance du troc dans leur société. Un mouton vaut quatre brebis ou un sir de beurre clarifié ou une couverture de cheval en poil de chèvre ou une pièce de feutre d'environ cinq mètres carrés. Un cheval vaut dix à douze moutons. Un chameau, au minimum vingt-cinq moutons. Chez les Warhis, sédentaires, le blé et l'orge servent de référence : le mouton vaut vingt sirs de grain ou trois de riz ou cinq mètres de velours côtelé ou deux à trois kilos de thé. Les autres marchandises régulièrement échangées sont le sel, le qurut (yogourts séchés), les fers à cheval et l'opium. L'argent liquide est très peu utilisé, sauf dans les bazars.

Nous avons donc été intégrés à la caravane de chameaux. Nous montions des chevaux mais n'étant pas cavaliers, cela nous était dur, très dur. Trois chevaux supplémentaires portaient nos bagages.

Je porte autour de la taille, dans une banane, seulement deux objectifs, un de $90 \mathrm{~mm}$, l'autre de 180 , et ne garde en permanence autour du cou qu'un appareil équipé d'un $35 \mathrm{~mm}$. J'ai donc trois objectifs à ma disposition.

Engoncé dans mes vêtements, j'éprouve une évidente gêne à monter et descendre de cheval sans l'aide d'un caravanier à qui je dois confier ma monture pour être libre de mes mouvements.

En dépit du froid, pour opérer les réglages - ouverture du diaphragme, vitesse d'obturation, profondeur de champ - et pouvoir appuyer sur le déclencheur, j'enlève mes gants et ne garde que des sous-gants en soie. Concernant la prise de vue proprement dite, il faut anticiper l'emplacement le plus approprié au cadrage. J'ai trouvé l'endroit. Je suis là avec la caravane : je vais m'arrêter, je les laisse partir. Il y a du vent. La température descend jusqu'à $-20^{\circ}$. La caravane suit, sur les berges, les méandres de la rivière. Elle la traverse de temps à autre. Du matin au soir, elle ne s'arrête, jamais mais elle avance lentement. J'attends avec mon cheval qu'elle soit éloignée pour réaliser la prise de vue, puis je la rattrape.

Chaque fois que je change de pellicule en ouvrant le boîtier, réchauffée par mon haleine, des gouttelettes d'eau tombent en stalactites de ma barbe gelée et se solidifient immédiatement sur le rideau de l'obturateur. Ces gouttelettes, telles des larmes, ajoutent à mon accablement en me faisant craindre soit des désordres dans l'image, ou pire, sa disparition. La nuit, nos appareils dorment avec nous dans nos sacs de couchage afin que les piles de nos cellules ne gèlent pas, mais nous nous demandons chaque jour si ce sera suffisant.

Aujourd'hui, j'ai eu envie de saisir de loin la silhouette noire des dix-sept chameaux de la caravane se découpant en file indienne sur la rivière gelée (fig. 19). Nous nous trouvions à quatre mille mètres d'altitude, il y avait encore trois mille mètres de dénivelé au-dessus de nous. Utilisant mon téléobjectif de $180 \mathrm{~mm}$, qui permet de tasser davantage les montagnes et de leur donner plus de hauteur, je gomme littéralement le ciel. Tout se fond dans une dominante de camaïeux gris-mauve, gris-bleu et gris de cendre. Je voudrais que cette photo soit le pendant visuel de l'image littéraire de Malraux. Mais sera-t-elle réussie?

Elle le sera par une sorte de petit miracle, car capter une émotion intense par le biais d'une photo reste la chose la plus difficile au monde, même si l'on sait que le mot « image » est l'anagramme de «magie».

Puis nous quittons la rivière. Malgré le soleil et le ciel bleu, la température est très basse. Seuls les chameaux ne semblent pas être incommodés. Tels des seigneurs imperturbables, magnifiques, ils progressent silencieusement en ramassant d'un coup de langue de vastes lampées de neige, à défaut de Bellum Tataricum (herbe à chameaux), cette rhubarbe sauvage dont ils sont si friands, mais qu'ils ne retrouveront qu'au printemps. Les chameliers, les oreillettes de leur coiffure rabattues sur le front et les oreilles, le visage enfoui dans leur col de fourrure, n'échangent pas une parole, chacun fait provision d'énergie pour lutter contre le froid.

Je parle rarement de la façon dont je fais les photos. Pourtant, souvent, au moment de nous endormir, nous nous posons la question : «Nos photos seront-elles réussies? » Notre but étant de devenir photographes, il était capital, pour nous, de réaliser des photos de qualité.

10. Entretien avec Roland et Sabrina Michaud, avril 2014. 


\section{Le carnet de 1982}

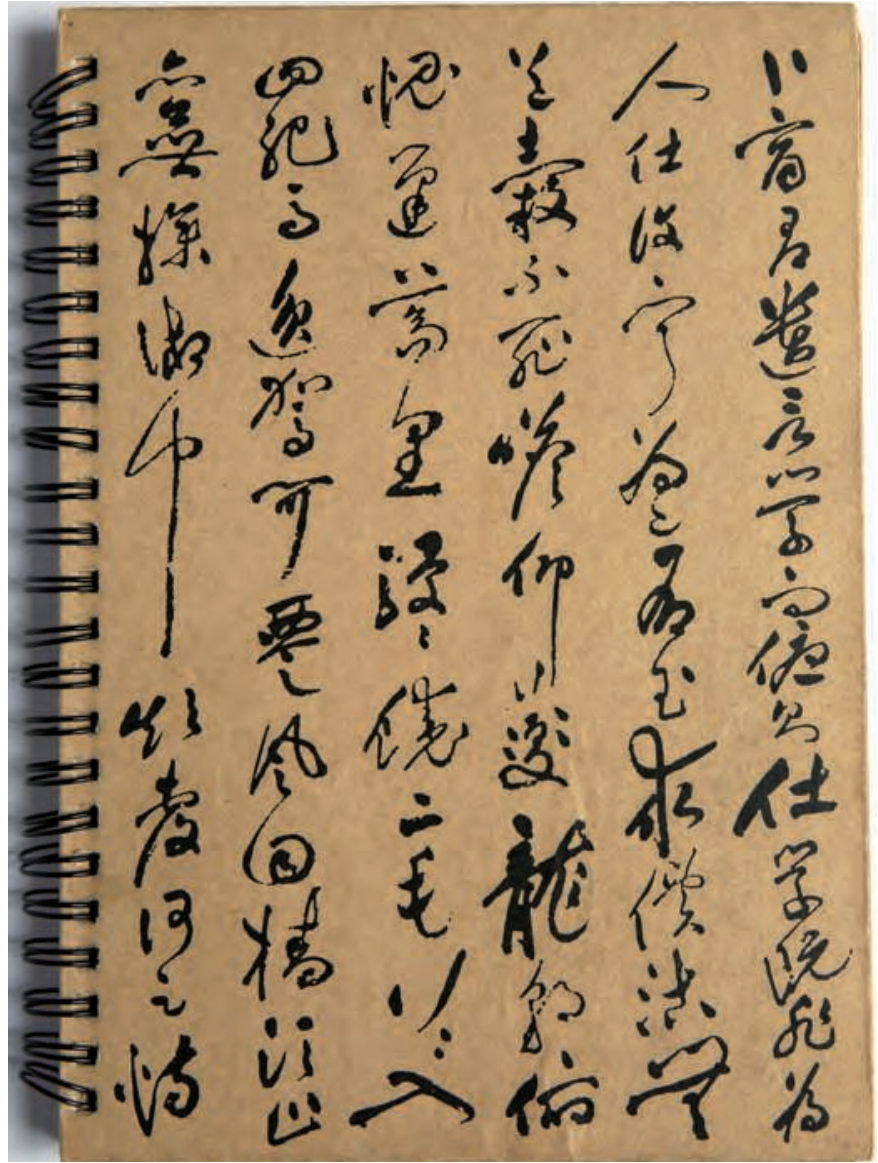

Fig. 21 : Couverture et fo 59 du carnet brun acheté en Chine lors d'un voyage ultérieur fut utilisé plus tard, en 1982, par Roland Michaud, qui tente alors de rassembler ses « souvenirs de la caravane d'hiver sur le toit du monde »

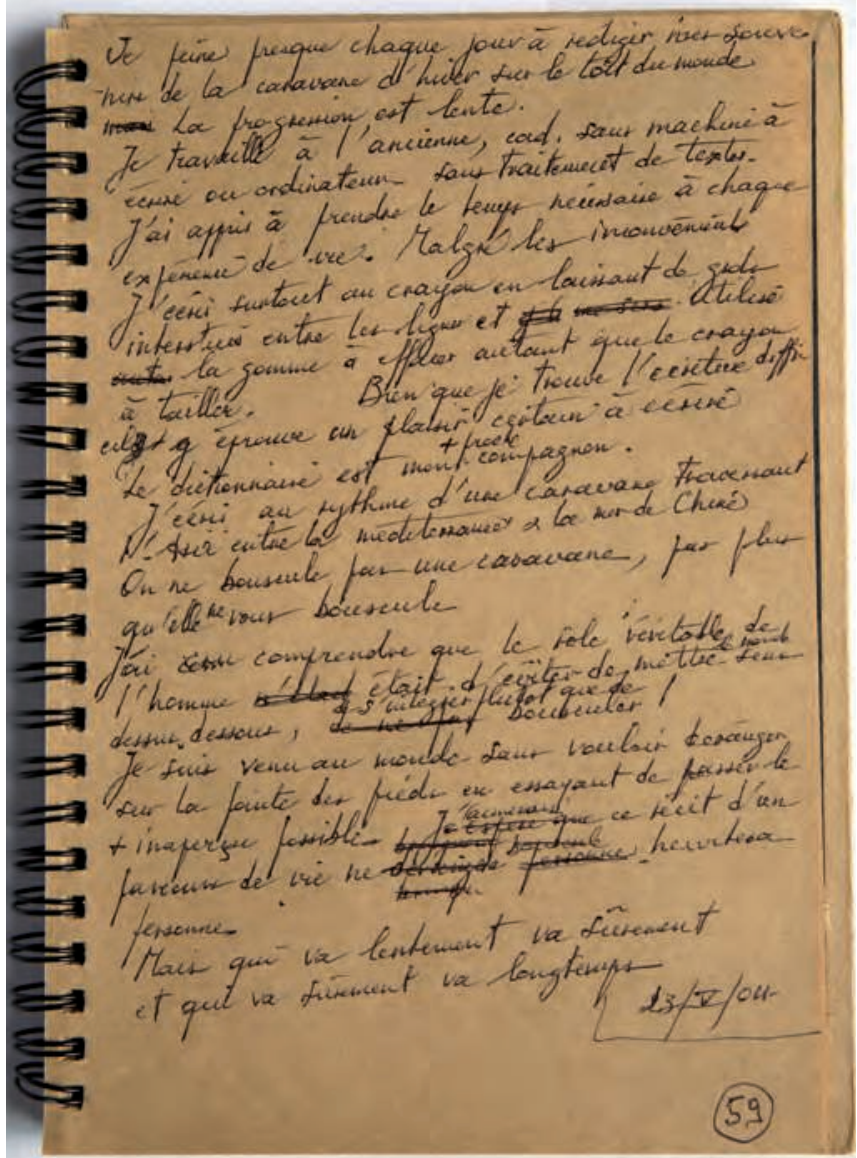

« Je peine presque chaque jour à rédiger mes souvenirs de la caravane d'hiver sur le toit du monde. La progression est lente. Je travaille à l'ancienne, cad, sans machine à écrire ou ordinateur - sans traitement de textes. J'ai appris à prendre le temps nécessaire à chaque expérience de vie. Malgré les inconvénients. J'écris surtout au crayon en laissant de grds interstices entre les lignes et je me sers utilise auta la gomme à effacer autant que le crayon à tailler. Bien que je trouve l'écriture difficile j'éprouve un plaisir certain à écrire. Le dictionnaire est mon + proche compagnon. J'écris au rythme d'une caravane traversant l'Asie entre la Méditerranée et la Mer de Chine. On ne bouscule pas une caravane, pas plus qu'elle ne vous bouscule. Jai cru comprendre que le rôle véritable de l'homme n'était était d'éviter de mettre le monde sens dessus dessous, de s'interroger plutôt que de bousculer. Je suis venu au monde sans vouloir déranger, sur la pointe des pieds en essayant de passer le + inaperçu possible. J'espère aimerais que ce récit d'un parcours de vie ne brusquera dérangera brusqu bousetle heurtera personne. Mais qui va lentement va sûrement et qui va sûrement va longtemps. »

Cet article a été produit dans le cadre du programme de recherche Photopaysage (ANR-13-BSH3-0008-01). 


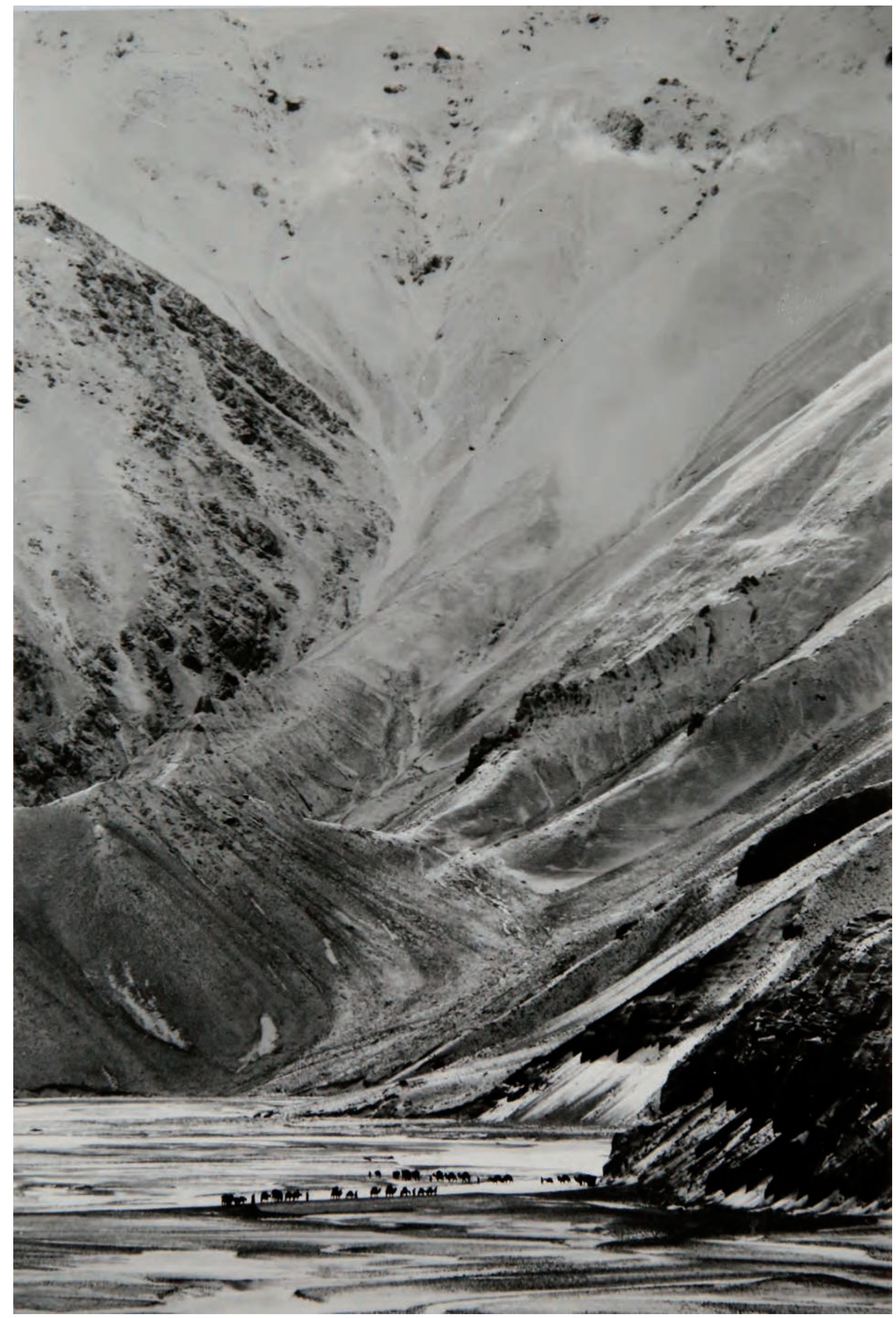

Fig. 22 : Tirage noir et blanc réalisé d'après un Kodachrome : la caravane de chameaux sur la rivière gelée du Warhan 